\title{
Fiber Arrangement of the Rat Splenic Capsule with Special Reference to Elastic Fibers
}

\author{
By \\ Tamotsu KAI, Hisanori NISHIZONO and Kazuo KIMURA

\begin{abstract}
Department of Anatomy (Director Prof. M. MIYOSHI), Fukuoka University School of Medicine, Fukuoka 814-0180, Japan
\end{abstract}

- Received for Publication, September 2, 2000 -

Key Words: Rat splenic capusule, Elastic lamina, Extravascular lymph passways, Fine structures

\begin{abstract}
Summary: The fiber arrangement of the rat splenic capsule was examined under scanning electron microscope (SEM) after selective removal by chemical maceration of capsule components, in conjunction with examination of thin sections using a transmission electron microscope (TEM). TEM findings were compared to those of the guinea-pig, mouse and dog.

Serosal cells of the rat splenic capsule showed labyrinthic channels that consisted of expanded intercellular spaces and tunnel-like spaces or slits in the basal cytoplasm. The channels contained fascicles of collagenous fibrils, forming a loose net on an underlying elastic lamina of densely aggregated elastic fibers. The fibrous tunica was an intermingled layer of elastic and collagenous fibers and sparse interstitial cells. The fibrous components were arranged more densly in the external layer than in the internal layer of the capsule. The elastic lamina comprised the outermost layer of the fibrous tunica in the intertrabecular region, but was situated deep in the trabecular region. Outward branches of the lamina in the trabecular region were arranged in a spiral. Intrachannel fibrillar fascicles were continuous, via fenestrations in the elastic lamina, with thick fascicles of collagenous fibers. The elastic lamina may play a role in effective contraction of whole spleen. In the guinea-pig, mouse and dog, elastic fibers were fragmented and not arranged in laminae. Typical smooth muscle cells were abundant in the dog.

The channel spaces in the serosal tunica are discussed from the viewpoint of extravascular lymphatic flow for circulation of the peritoneal serous fluid.
\end{abstract}

The contractile elements such as smooth muscle cells and elastic fibers in the splenic capsule and its trabecules play an important role in the outflow of pooled blood (Raviola, 1986, Amenta and Amenta, 1990). As summarized in detail by Tischendorf (1969), their volume development is inversely proportional and their arrangement varies with animal species. In laboratory rodents (guinea-pig, rat, mouse, rabbit) and humans, the spleen contain a relatively small blood volume and the musculature is poorly developed. Elastic fibers in the capsule of those animals are arranged in a three dimensional network throughout the capsular layer, being homogeneously intermingled with sparse smooth muscle cells and thick fascicles of collagenous fibers (Rodrigues et al., 1999). Furthermore, laminar structures of elastic fibers are also reported to be the limiting membrane in rabbit and mouse (Gross, 1951) and the lamina elastica (Porsio, 1932; Tischendorf, 1969) in the dog, which has a large volume of blood in the spleen. Our preliminary examination of the rat splenic capsule showed the presence of an elastic lamina (Nishizono, Miyoshi and Kai, 1994). However, detailed information is not available on the structural relationship between the laminar elastic fibers and other capsular components.

On the other hand, the overlying serosal tunica is known to share the metabolic function of the abdominal serous fluid (Andrews and Porter, 1973; Tanikawa, 1979). Serosal cells lined with a loose fibrous net are generally thought to have a high active absorptive function (Fawcett. 1986; Oya, Shimada, Nakamura and Uchida, 1992). However, no characteristic structures have been reported of the serosal tunica associated with a dense layer of connective tissue to our knowledge.

Our aim in this study was to clarify three dimensional arrangement of fibrous elements, particularly the elastic lamina, under SEM after selective preservation of elastic fibers and collagenous fibers in the rat splenic capsule, and to compare it to the 
fine structures of the whole capsule layer by TEM observation in thin sections. The fine structural relationship between the laminar elastic fibers and the serosal tunica is discussed from the viewpoint of metabolic functions of the peritoneal serous fluid.

\section{Materials and Methods}

Thirty-two Wistar rats of both sexes weighing about $250 \mathrm{~g}$ were used. For comparative observation of fine structures, seven mice of about $50 \mathrm{~g}$, five ginea-pigs of about $250 \mathrm{~g}$, and three dogs in about $9 \mathrm{~kg}$ were also used. The animals excluding dogs were anesthetized by inhalation of ether. The dogs were anesthetized by intraperitoneal injection of $25 \mathrm{mg}$ pentobarbital- $\mathrm{Na} / \mathrm{kg}$ weight. Each small animal was perfused via the descending aorta with 10 or $40 \mathrm{ml}$ Ringer's solution for rinsing out blood in vessels, and then, with 100 to $250 \mathrm{ml}$ of fixatives. Dog spleens were removed and perfused via the lienal artery with $50 \mathrm{ml}$ Ringer's solution and $250 \mathrm{ml}$ fixative. The main fixative used was $2.5 \%$ glutaraldehyde in $0.1 \mathrm{M}$ phosphate buffer adjusted to $\mathrm{pH}$ 7.4. The $10 \%$ paraformaldehyde in $0.1 \mathrm{M}$ cacodylate buffer adjusted to $\mathrm{pH} 7.4$ was used for SEM specimens of elastic fibers (Ushiki, 1992). All perfused spleens were kept in the same fixative for one week at room temperature.

For TEM observation: Glutaraldehyde-fixed specimens were cut into small pieces, post-fixed with $1 \%$ osmium tetroxide in $0.1 \mathrm{M}$ phosphate buffer adjusted to $\mathrm{pH} 7.4$, dehydrated through a series of graded ethanol and propylene oxide, and were embedded in Epon epoxy resin. Thin sections of plastic-embedded specimens were made, and stained with uranium nitrate and lead citrate. Some sections were immersed in tannic acid solution to stain the elastic fibers before metal staining.
(Watanabe and Nishizono, 1994) Observation was performed with a Hitachi $\mathrm{H} 7000$ type TEM.

For SEM observation: (1) Specimens of the serosal tunica were prepared from glutaraldehydefixed materials. Tissue blocks with observing surfaces (about $3 \times 3 \times 2 \mathrm{~mm}$ ) were made with razor blades. After desiccation, the observing surfaces of some tissue blocks were blown with air jets to partially remove the serosal tunica. (2) Collagenous fibers were exposed by immersion of tissue blocks $(3 \times 3 \times 2 \mathrm{~mm})$ in $10 \%$ sodium hydroxide for 5 days at $20^{\circ} \mathrm{C}$ (Ohtani, 1987) and, were thoroughly washed in several changes of distilled water for 5 days at $20^{\circ} \mathrm{C}$. (3) Elastic fibers were exposed by immersion of tissue blocks (about $3 \times 3 \times 2 \mathrm{~mm}$ ) of paraformaldehyde-fixed materials in $90 \%$ formic acid for 4 days at $45^{\circ} \mathrm{C}$ (Ushiki, 1992), and rinsed with Mcllvane buffer adjusted to $\mathrm{pH} 3$. All tissue blocks mentioned above were immersed in $0.1 \%$ aqueous solution of tannic acid (for the elastic fiber, $0.1 \%$ tannic acid in McIlvane buffer mentioned above) for $0.5 \sim 1 \mathrm{hr}$, washed in several changes of distilled water, and were post-fixed with $1 \%$ osmium tetroxide in phosphate buffer mentioned above for $2 \mathrm{hrs}$. The post-fixed specimens were rinsed with distilled water, dehydrated through a series of graded ethanol, placed in t-butylalcohol, and desiccated by the frozen-dry method. After fixation of dried specimens on metal blocks, observing surfaces were coated with gold-palladium alloy in an evaporator. Observation was made with a Hitachi S-450 type and a S-900 type SEM.

\section{Observations}

TEM observation: The serosal tunica in the rat was a single layer of squamous serosal cells (Figs. 1, 2). The swollen perikarya contained some dense

\section{Explanation of Figures}

\section{Plate I}

Survey TEM views of the whole layer of a rat splenic capsule.

Fig. 1. A intertrabecular region. Flattened serosal cells are separated by a thick elastic lamina (EL) with sporadic pores (arrowhead) from a underlying fibrous tunica (FT), which contains interstitial cells (IC), fragmented elastic fibers (arrow with E) and thick fascicles of collagenous fibers (C). Slits and tunnel-like spaces (arrows) are formed between the elastic lamina and serosal cells (SC) S:venous sinus. $\times 4,400$

Fig. 2. A trabecular area. At deep level is situated an elastic lamina (EL) with many large pores, through which fascicles of collagenous fibers (C) and interstitial cells (IC) extend. Serosal intercellular spaces (IS) with cell junctions (small arrows) form large lucent spaces. $\times 4,400$

Fig. 3a. Basal slits and cytoplasmic cavities contain fascicles of collagenous fibrils (CF) and their cytoplasmic membrane is associated with a basal lamina (arrowheads). EL:elastic lamina. $\times 8,000$

Fig. 3b. Intercellular spaces (IS) are sealed by cell junctions (small arrows) and their basal wall is open (large arrow). $\times 8,000$ 

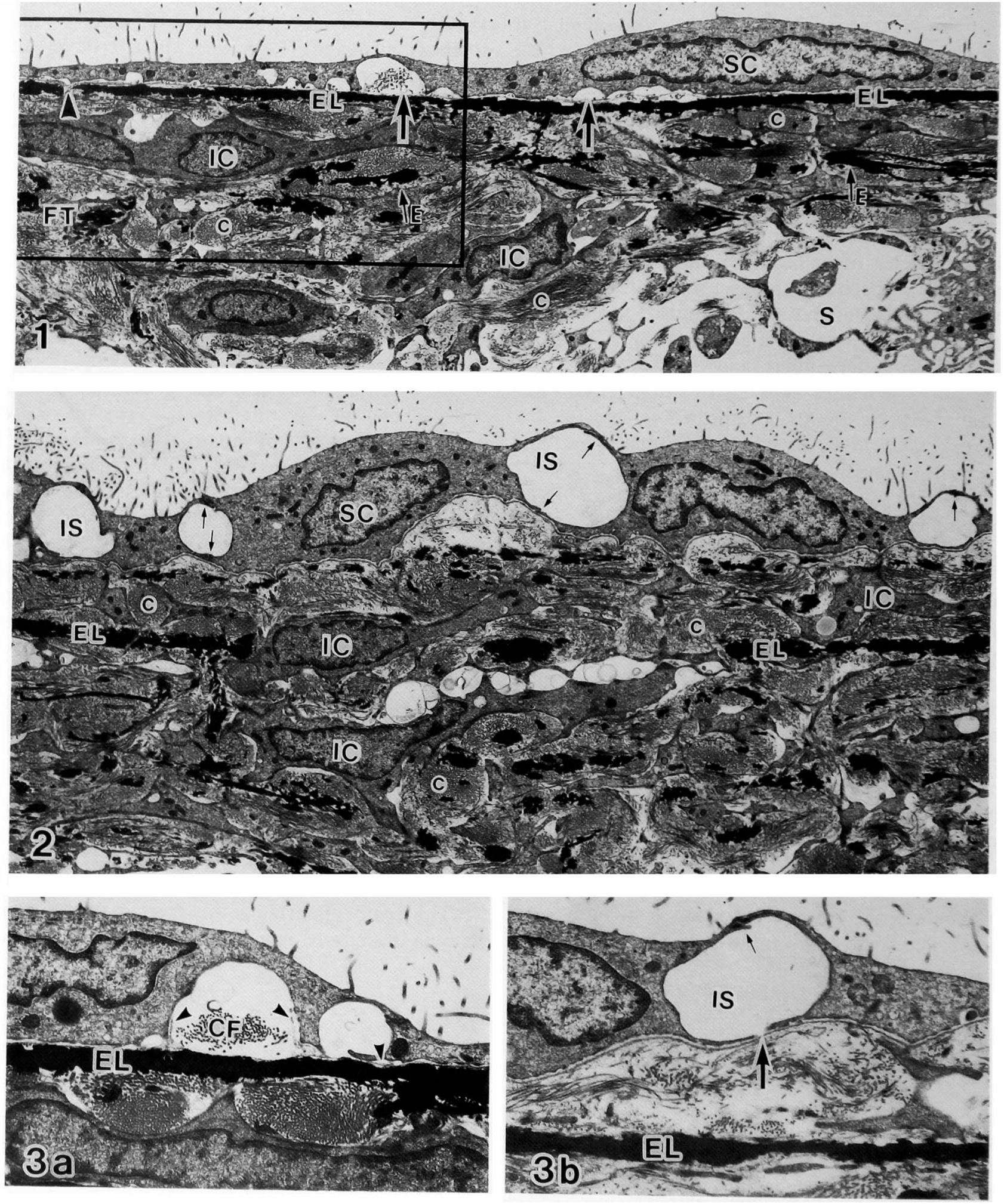
large granules, mitochondria and granular endoplasmic reticulum around a flattened nucleus. Junctional structures were seen between neighboring cells. Finger-like microprojections were well developed on free cell surfaces. In intertrabecular regions, lucent spaces were conspicuous in the basal cytoplasm of serosal cells (Figs. 1, 8a, 8b), which were variably sized tunnel-like spaces (about $2 \mu \mathrm{m}$ or less in diameter) and slits. The spaces communicated each other, forming labyrinthic channels. A delicate basal lamina (about $10 \mathrm{~nm}$ in thickness) was seen on the cell membrane that was lining the basal surface or channel spaces (Fig. $1,3 a, 8 a, 8 b$ ). The channel spaces frequently contained variably sized fascicles of collagenous fibrils. The channel spaces in trabecular regions usually developed as expanded intercellular spaces (up to $5 \mu \mathrm{m}$ in diameter) between neighboring serosal cells (Figs. 2, 3b). Those intercellular spaces were sealed by junctional structures (Fig. 2) and their basal cytoplasm was frequently perforated (Figs. 2, 3b). However, openings of the intercellular space into the peritoneal cavity were not observed.

The fibrous tunica was composed of intermingled fascicles of collagenous fibers, elastic fibers and a small number of interstitial cells (Figs. 1). Those cells with larger cytoplasmic processes were stellate and elongated along the capsule (Figs. 1, 8a, $8 b)$. Their cytoplasm contained fascicles of thin cytoplasmic filaments (about $20 \mathrm{~nm}$ in thickness), numerous ectoplasmic caveolae, and some small membrane-bound granules. The fibrous tunica in intertrabecular regions was different in structures from that in trabecular regions (Figs. 1,2). In the intertrabecular regions, elastic fibers formed a continuous lamina (about $0.5 \mu \mathrm{m}$ in thickness) with sporadic fenestrations of varying sizes (from 1 to $0.5 \mu \mathrm{m}$ in diameter) (Fig. 1, 8a, 8b). The lamina was situated just beneath the serosal tunica. In the trabecular region, however, it seemed to be a sieve membrane with many large fenestrations situated deep in the fibrous tunica (Figs. 2, 3b). Therefore, those fenestrations contained fascicles of collagenous fibers and cytoplasmic processes of interstitial cells.

Structural components of splenic capsules in the mouse and guinea-pig were fundamentally similar to those in the rat (Figs. 4, 5). Elastic fibers were fragmented and homogeneously intermingled with fascicles of collagenous fibers and interstitial cells. Intraserosal channel spaces were expanded intercellular spaces in the mouse (Fig. 4). They were tunnel-like in the basal cytoplasm and tubular or vesicular in the cytoplasm in the guinea-pig (Fig. 5). The development of the spaces in the guinea-pig varied by serosal cells. The dog splenic capsule was thick and contained numerous smooth muscle cells with typical cytoplasmic structures (Fawcett, 1986) and a small amount of fragmented elastic fibers among thick fascicles of collagenous fibers (Fig. 6).

SEM observation: The serosal tunica of the rat splenic capsule in unmacerated specimens was comprised of a sheet of squamous serosal cells (Fig. 7). The perikarya were swollen with discoidal plates of nuclei, and the free cell surfaces showed numerous finger-like projections. The serosal cells in the intertrabecular regions were lined by a plate. Partial removal of the serosal tunica showed a rough net of variably sized fascicles of collagenous fibrils (about $2 \mu \mathrm{m}$ or less in thickness) and their extensions beneath serosal cells or into fenestrations of the underlying plate (Fig. 7).

The selective preservation of fibrous components showed characteristic surface structures: branching elastic fibers with a typical rough surface caused by a parallel arrangement of rodlets of similar size (about $60 \mathrm{~nm}$ in thickness) (Fig. 9b) and collagenous fibers in fascicles of smooth fibrous components in uniform thickness (about $50 \mathrm{~nm}$ ) (Fig. 12b).

The plate underlying the serosal tunica was a lamina of densely aggregated elastic fibers and was

Plate II

Comparative TEM views of the whole layer of intertrabecular splenic capsules in two species of rodents and one species of carnivore.

Fig. 4. A mouse splenic capsule. A relatively thick elastic lamina (EL) separates the fibrous tunica into supra-and infralaminar areas. Both areas are occupied by intermingled fascicles of collagenous fibers (C), fragmented elastic fibers (arrow with E), and interstitial cells (IC). The serosal tunica contains large lucent spaces (CS) between neighboring serosal cells $(\mathrm{SC}) . \times 4,400$

Fig. 5. A guinea-pig splenic capsule. Structural components of the fibrous tunica are quite similar to those in the mouse (Fig. 3 ). However, the fascicles of collagenous fibers (C) are relatively large. Fragmented elastic fibers (arrows with E) and flattened interstitial cells (IC) are sparse. Serosal cells (SC) contain poorly developed tubular spaces (arrows). L:lymph capillary. $\times 4,400$

Fig. 6. A dog splenic capsule. A thick fibrous tunica comprises interlacing robust fascicles of collagenous fibers (C), fragmented elastic fibers (arrows with E) and smooth muscle cells (SM) with typical cytoplasmic structures and elongated processes. Serosal cells (SC) with numerous microvilli are associated with a distinct basal lamina (BM) and small numbers of lucent cytoplasmic spaces $(\mathrm{CS}) . \times 4,400$ 

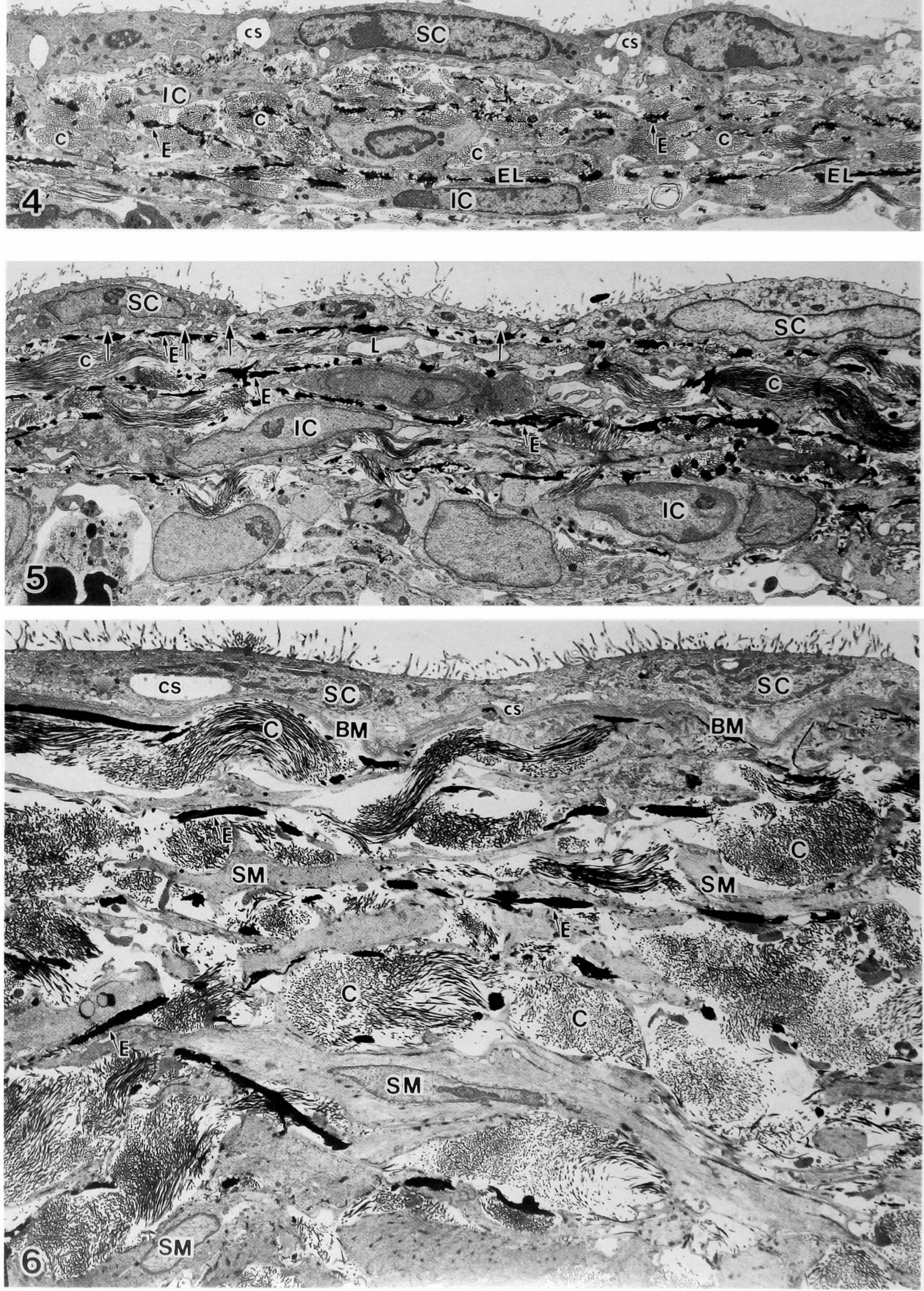
fenestrated with sporadic pores of varying sizes (about 1 to $0.5 \mu \mathrm{m}$ in diamether) (Fig. 9a). The fibers in the lamina were branched and wound or curled, especially around fenestrations. The elastic lamina in trabecular regions was fenestrated with relatively large pores and showed outward branches that were arranged in a whirlpool (Fig. 10).

In the deep layer of the fibrous tunica, elastic fibers of various thickness (from $0.5 \mu \mathrm{m}$ to $50 \mathrm{~nm}$ ) were arranged in a three dimensional network (Fig. 13).

Collagenous fibers were fasciculated at varying thickness (from about 10 to $0.5 \mu \mathrm{m}$ ). The fascicles just beneath the elastic lamina were densely interlaced, forming a layer with a smooth external surface in the intertrabecular regions (Fig. 11) and with a rough surface with napped fibrils in the trabecular regions (Fig. 12a). Fascicles of collagenous fibers of varying thickness (from 2 to $1 \mu \mathrm{m}$ ) in the internal layer of the capsule also constituted a rough three dimensional network (Fig. 14). Fascicular surfaces and meshes of the networks were covered with irregular delicate collagenous fibers.

\section{Discussion}

Selective preservation of the elastic and collagenous fibers in this study showed the three dimensional arrangement of each fiber under SEM. The tannic acid selectively stained elastic fibers in thin sections.

Our findings from TEM and SEM study indicate that the intermingled structural components of the fibrous tunica are in a three dimensional network, and that the elastic fibers and fascicles of collagenous fibers are arranged densely in the external layer and loosely in the internal layer. The elastic lamina reported by Porsio (1932) in the dog is developed in the rat but has not been observed in the dog in this study. Furthermore, the development of elastic fibers was poor in the dog, whereas smooth muscle cells were abundant. The reasons for our failure to find the elastic lamina are not presently known, but it may be due to a difference in the breed of dog examined. However, our findings support the general concept that spleens with a large volume of blood (ruminants, carnivores etc.) have poor elastic fibers, instead of a robust connective tissue and muscular framework (Tischendorf, 1969; Raviola, 1986).

The elastic lamina in the rat is a complete sheet of densely aggregated elastic fibers. Therefore, it may be considered that the contraction of the elastic lamina is quite effective to squeeze out pooled blood evenly from all parts of the spleen. Furthermore, the lamina extends inwards sending threads of elastic fibers into intertrabecular regions, and also outwards to trabecular regions. The elastic lamina in the trabecular region was separated from the serosal tunica by a loose connective tissue layer. Therefore, it may correspond to the limiting membrane in other species of rodents (mouse and rabbit) (Gross, 1951).

Our findings showed channels between neighboring serosal cells and in their basal cytoplasm. Although the spaces became more obvious with perfusion as in the intestinal epithelium (Ogawa and Miyoshi, 1984), they demonstrate characteristic structures. Intercellular channel spaces usually occurred in serosal tunicae with an underlying loose fibrous net. The spaces were sealed by cell junctions, but their basal cytoplasm was frequently open toward the basement membrane. However, tunnel-like spaces and slits in the basal cytoplasm were seen in serosal tunicae with subjancent elastic laminae. These spaces were considered to be extraepitheliar, because they usually contained fascicles of collagenous fibrils and their cell membrane was lined by a delicate basal lamina. The intercellular spaces and tunnel-like spaces or slits may possibly communicate as labyrinthic channels. The channel system may be for extravascular lymphatic flow (Oya et al., 1992) for effective exchange of serous fluid through the serosal tunica. Fascicles of

\section{Plate III}

Capsule surface of a rat spleen and its TEM images.

Fig. 7. A SEM photograph of the serosal surface. The lower half is an exposed subserosal layer after serosal cells were removed by air jet. The serosal cells (SC) with discoidal nuclei are equipped with numerous microvilli. The exposed subserosal layer (SL) is associated with an irregular net of fascicles of collagenous fibrils (CF). Those fascicles extend beneath the serosal cells (arrows) and into holes (large arrow) of the subserosal layer. $\times 2,700$

Fig. 8a. A closeup of the squared portion in Fig. 1. Fascicles of collagenous fibrils (CF) are contained in basal cytoplasmic channels of serosal cells and extend through pores (large arrowheads) of the lamina elastica. $\times 6,800$

Fig. 8 b. Fascicle (CF) in a basal slit extends into a pore (large arrow) of the elastic lamina, the matrix of which is not stained with tannic acid and pale. $\times 6,800$ 

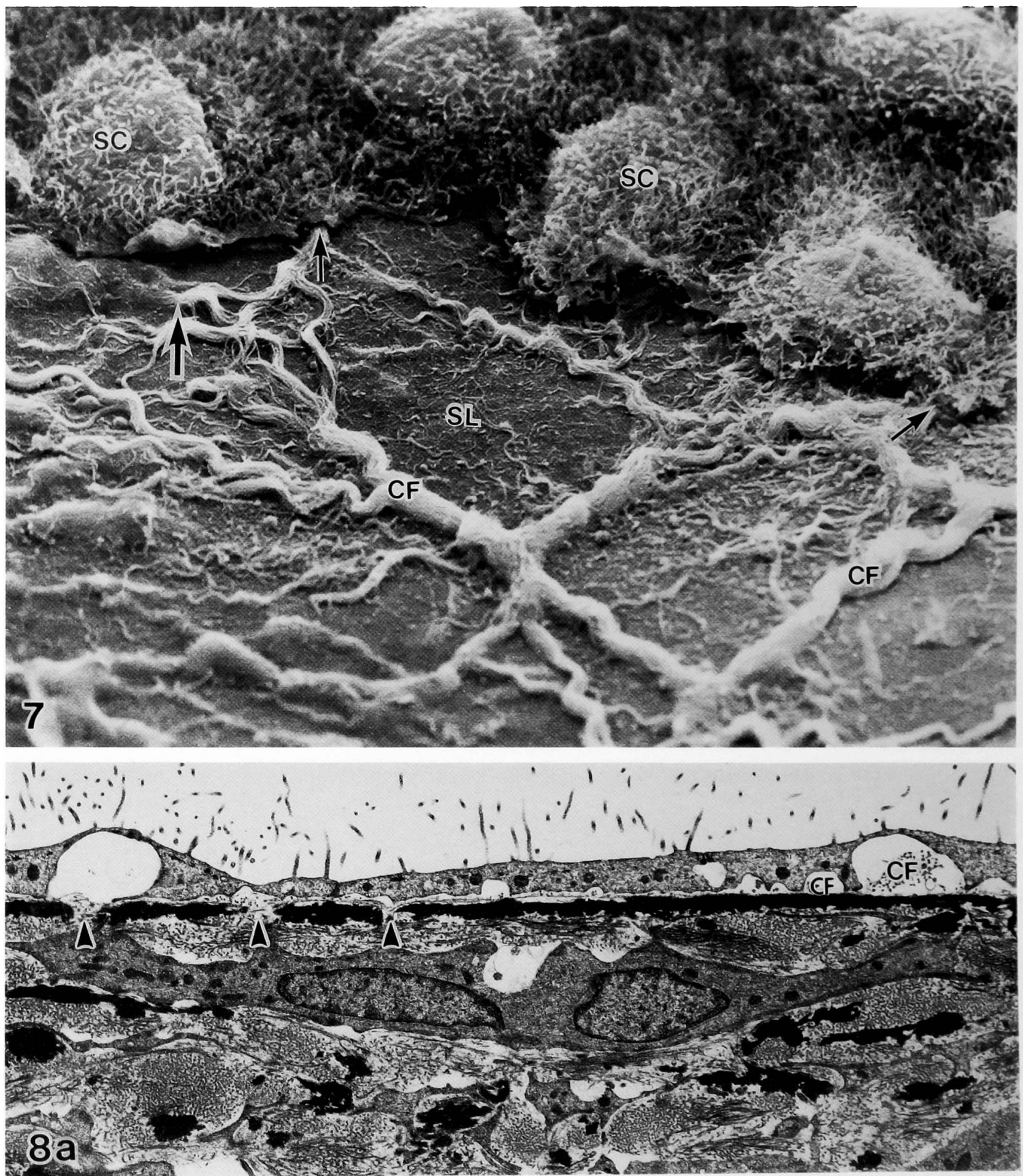

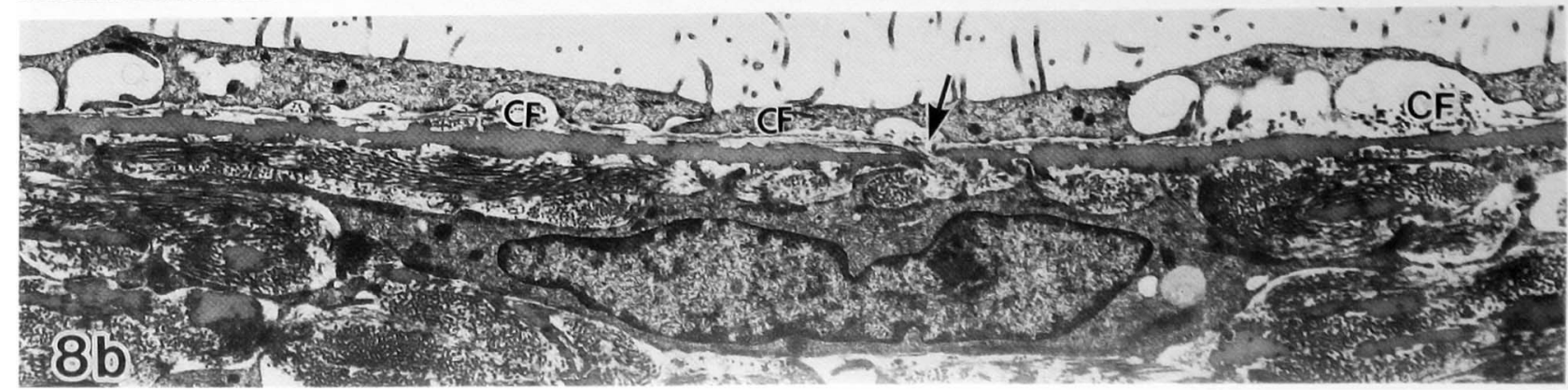


collagenous fibrils in the channel spaces were continuous via fenestrations in the elastic lamina with interlaced fascicles of collagenous fibers, and may play a role in fibrous drainage.

\section{Acknowledgements}

The authors are thankful to Ms. Takami Fusano for her help in TEM photography.

\section{References}

1) Amenta PS I and Amenta PS II. The anatomy of the spleen. In: (ed. by) Bowdler AJ: The spleen, Chapman \& Hall Medical, London, 1990 (p 3-7).

2) Andrews PM and Porter KR. The ultrastructural morphology and possible functional signification of mesothelial microvilli. Anat Rec 1973; 177:409-426.

3) Fawcett D. A text book of histology. Igakushoin/ Saunders Intnl, Tokyo, 1986: 660 .

4) Gross M. Zur Kentnis der Variationsbreite und des Feinbaues der normalen Milz von Laboratoriumratten. Med. Diss. Wurzburg, 1951.

5) Nishizono $H$, Miyoshi $M$ and Kai $T$. Fiber arrangement in the rat splenic capsule. In: Absract of XIV federative internat. congr. anat., Lisbon-Portugal, 1994 (p. 367).

6) Ogawa $\mathrm{K}$ and Miyoshi $M$. Intercellular spaces in the lyph- nodule associated epithelium of the rabbit Peyer's patches and appendix. Arch Histol Jpn 1985; 48:53-67.

7) Ohtani O. Three-dimensional organization of the connective tissue fibers of the human pancreas: A scanning electron microscopic study of $\mathrm{NaOH}$ treated-tissues. Arch Histol Jpn 1987; 50(5):557-566.

8) Oya $M$, Shimada $T$, Nakamura $M$ and Uchida Y. Functional morphology of the lymphatic system in the monkey diaphragm. Arch Histol Cytol 1992; 56:37-47.

9) Porsio A. Struttura della capsulla e dello stroma di alcuniorgani. Scritti Biol 1932; 7:69-100.

10) Raviola E. Spleen. In: (ed. by) Fawcett: A textbook of histology, Capt. 16 pp 464-478, Igakushoin/Saunders Intnl, Tokyo, 1986.

11) Rodrigues CJ, Sacchetti JC and Rodrigues AJ Jr. Agerelated changes in the elastic fiber network of the human splenic capsule. Lymphology 1999; 32:64-69.

12) Tanikawa K. The liver surface. In: Ultrastructural Aspects of the Liver and its Disorders. 2nd ed., pp 75-81, IgakuShoin, Tokyo New York, 1979.

13) Tischendorf F. Mikroskopiche Anatomie der Milz. In: Handbuch der mikroskopischen Anatomie des Menschen. Die Milz. Springer-Verlag, Berlin, Heidelberg, N. Y., 1969 VI/6 (p 158-471).

14) Ushiki T. Preserving the original architecture of elastin components in the formic acid-digested aorta by an alternative procedure for scanning electron microscopy. J Electron Microsc 1992; 41:60-63.

15) Watanabe $\mathrm{N}$ and Nishizono $\mathrm{H}$. A scanning and transmission electron microscope study of fiber arrangement in the hepatic capsule. Okajima Folia Anat Jpn 1994; 71:279-296.

\section{Plate IV}

SEM photographs of external surfaces of the subserosal layer of rat splenic capsule after maceration with formic acid.

Fig. 9a. The subserosal layer is a membranous layer of densely aggregated elastic fibers (E) of varying thickness. Sporadic occurrences of small pores (arrows) are seen through the layer. Fibers wind and curl (double arrows), especially around pores. $\times 4,300$. Inserted (Fig. 9b) is a high magnification of the subserosal layer. The surface is covered with similarly sized rodlets (arrowheads) that extend along the long axis of the fibers. $\times 24,000$

Fig. 10. Subserosal elastic lamina in the trabecular region shows many branches (small arrows) of elastic fibers that are arranged in a spiral. Large pores (large arrows) are also seen through the lamina. $\times 1,000$ 

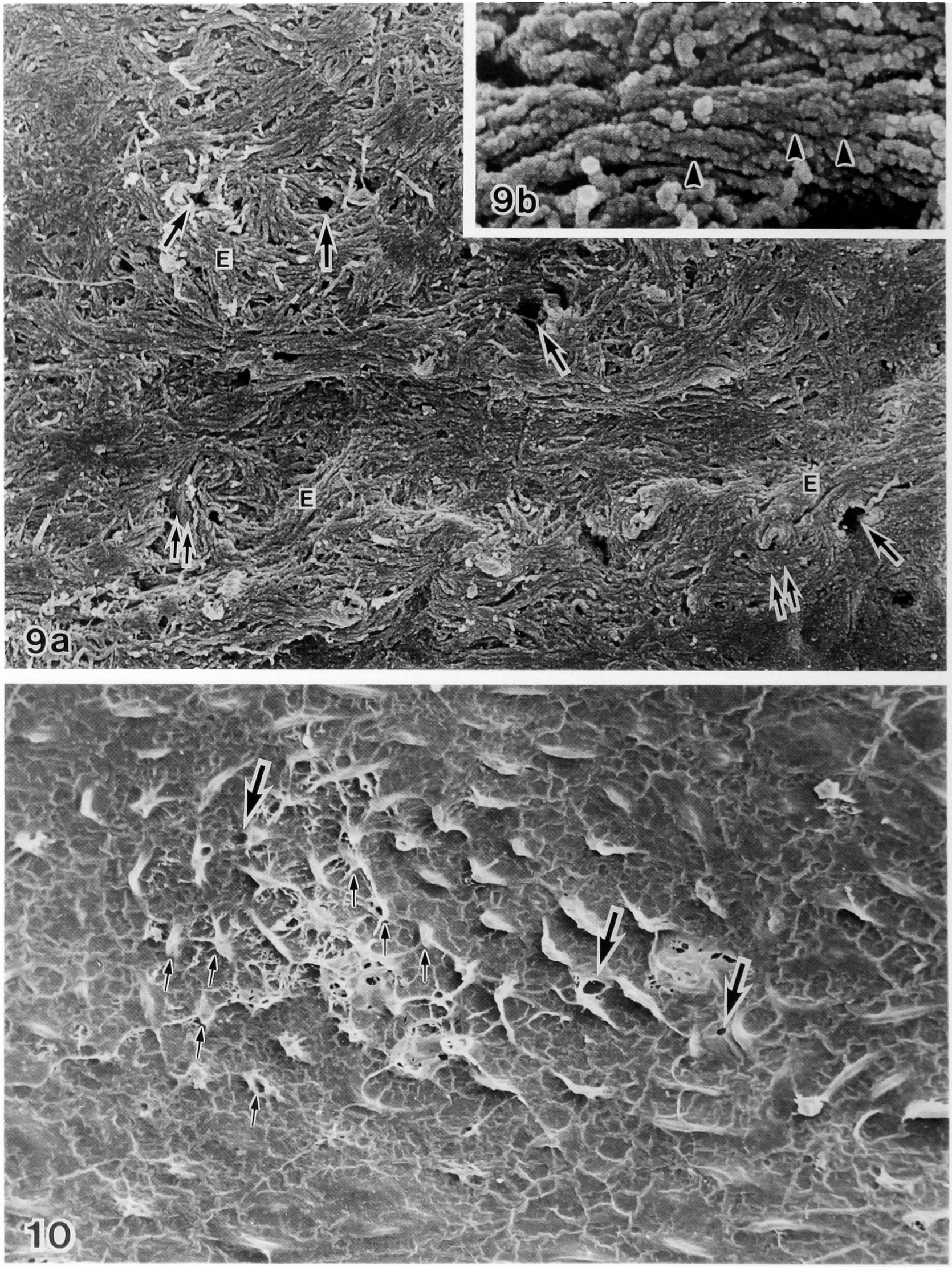


\section{Plate V}

SEM photographs of external surfaces of the rat splenic capsule after maceration with sodium hydroxide.

Fig. 11. Collagenous fibers are arranged in densely interlacing fascicles (C), which vary in thickness and anastomose with their branching fascicles. $\times 2,200$

Fig. 12a. Fascicles (C) of collagenous fibers are napped with collagenous fibrils. The corner figure (12b) is a high magnification of a fascicle $(\mathrm{C})$ of collagenous fibers (arrowheads) of uniform size. 12a: $\times 3,000,12 \mathrm{~b}: \times 13,000$ 


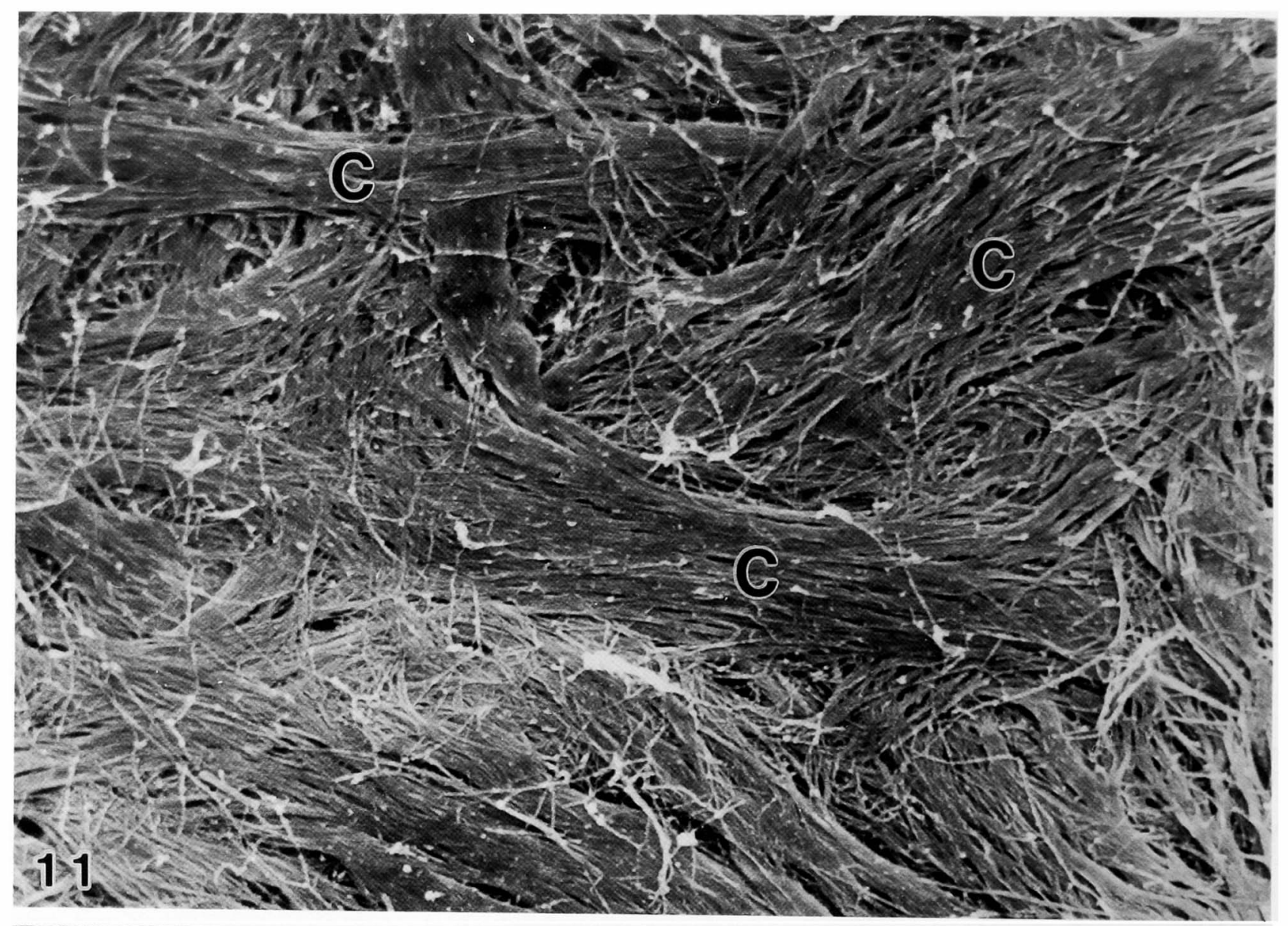

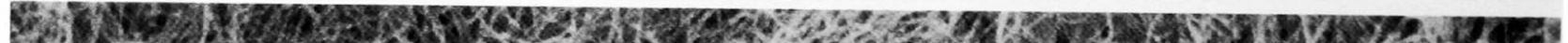

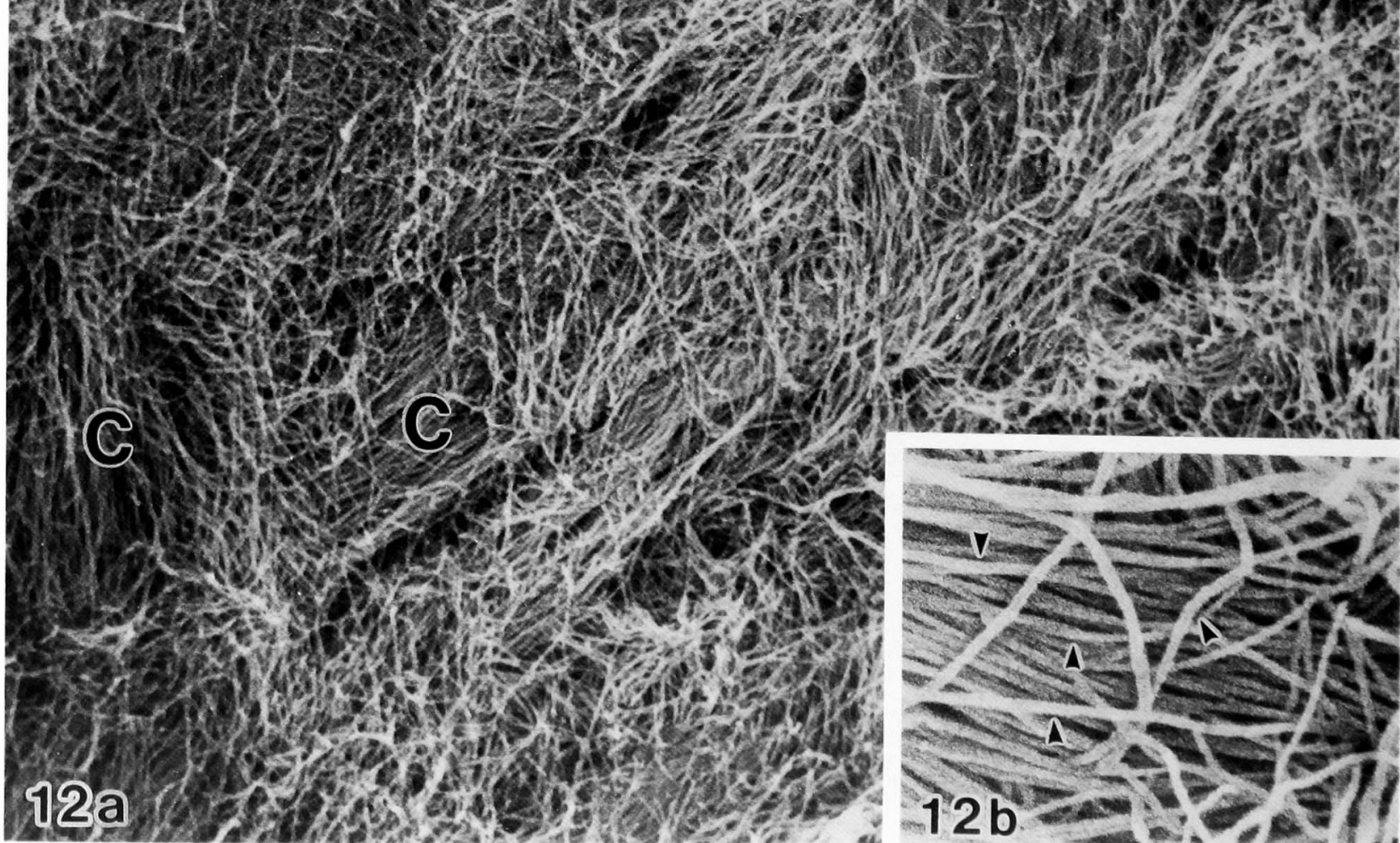




\section{Plate VI}

Internal surfaces of the fibrous tunica after maceration with formic acid (Fig. 13) and sodium hydroxide (Fig. 14).

Fig. 13. Elastic fibers (E) of various sizes are arranged in a three dimensional network by mutual anastomoses (asterisks) of branches. $\times 5,000$

Fig. 14. Napped fascicles of collagenous fibers (C) form a rough (asterisk) or dense meshwork (double asterisks) by their anastomoses. Meshes are filled with delicate collagenous fibers. $\times 2,000$ 

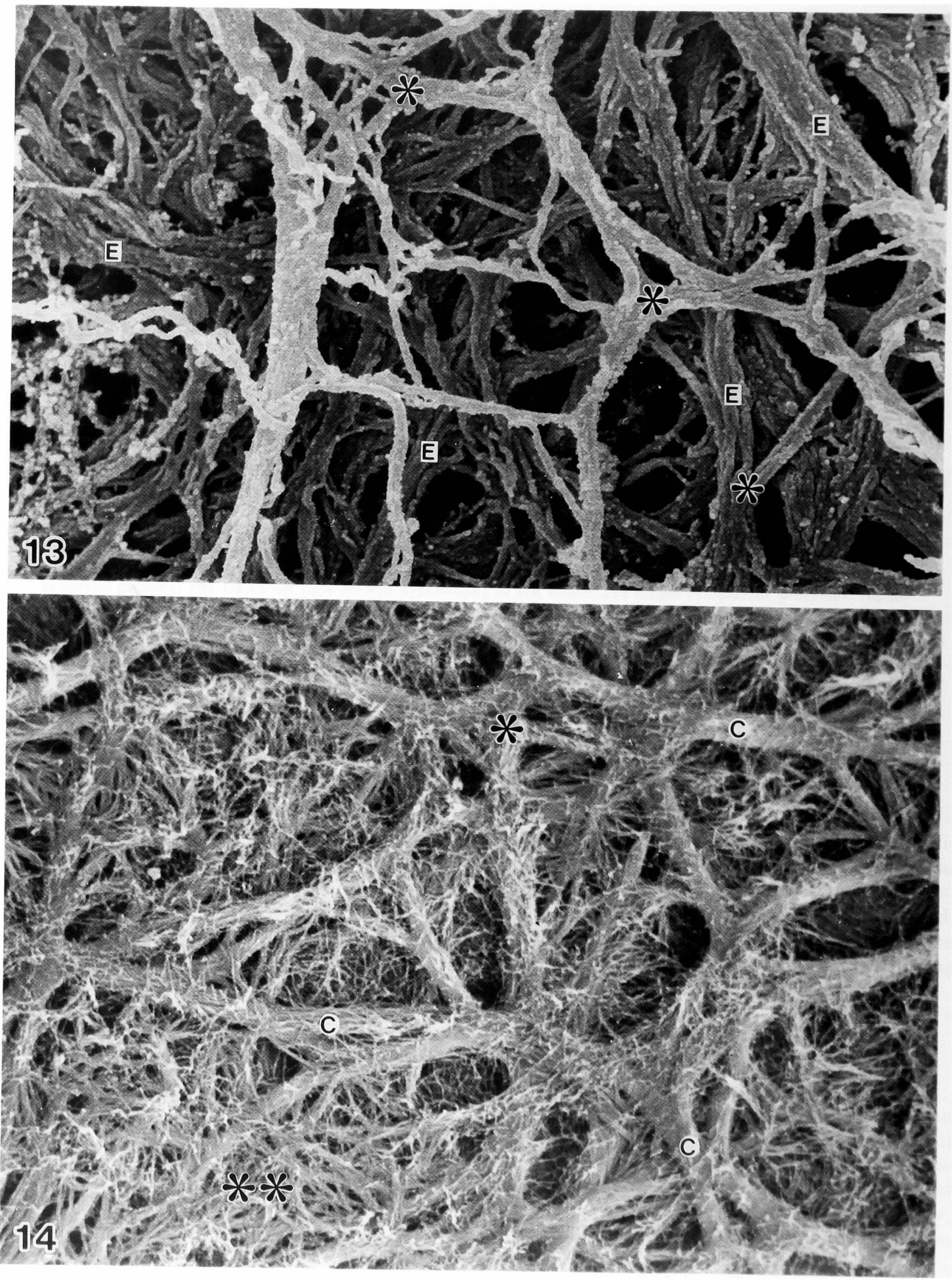